\title{
LOCATION-AWARE REAL-TIME SIMULATION FRAMEWORK FOR EARTHMOVING PROJECTS USING AUTOMATED MACHINE GUIDANCE
}

\author{
Faridaddin Vahdatikhaki \\ Shayan Setayeshgar \\ Department of Building, Civil, and Environmental \\ Engineering \\ Concordia University \\ 1515 Ste-Catherine Street West \\ H3G 2W1 Montreal, CANADA
}

\author{
Amin Hammad \\ Concordia Institute for Information Systems \\ Engineering \\ Concordia University \\ 1515 Ste-Catherine Street West \\ H3G 2W1 Montreal, CANADA
}

\begin{abstract}
The cost-and-time-optimized planning of earthmoving projects has been significantly boosted as a result of deploying simulation techniques which enable project managers to effectively comprehend the behavior of projects. However, the realism and accuracy of the simulation models diminish as a result of the heavy reliance on the statistical data and of not taking into account the context-specific features of the project. Similarly, the more unique the characteristics of projects and novel the construction methods, the less the possibility of retrofitting a historic pattern to new projects. On the other hand, the identification of potential accidents on construction sites has been a major concern in the construction industry. To address these issues, this research proposes a framework based on the integration of new tracking technologies used in Automated Machine Guidance (AMG) with a real-time simulation technique. A prototype is developed to test and demonstrate the effectiveness of the proposed approach.
\end{abstract}

\section{INTRODUCTION}

Although planning and optimization of small-size and some medium-size projects can rely on the professional intuition and experiences, large scale projects require a very meticulous and delicate planning, if productivity and efficiency are not to suffer. Therefore, in order to judge different scenarios for a given operation in a comparative fashion, and thus optimize the operation planning and resource allocation, it is indispensable to develop a digital model of operations, in terms of time-sequenced activities and the flow of resources. To this end, many researchers have investigated the application of simulation in the construction industry (AbouRizk 2010; Hassan, Al-Hussein, and Gillis 2010; Zayed and Halpin 2001; Hassan and Guber 2008; Halpin and Martinez 1999). However, simulation relies mainly upon the statistical data gathered from previous projects of the like nature. Given the volatility and dynamism of a construction system and the high sensitivity of short-term schedules to variations of constituent parameters, very often it is the case that the initially developed model fails to remain valid and representative of the actual work. To compensate for the possible discrepancies and deviations from the estimated values, i.e. time and cost, and also in order to make simulation results more realistic, the assumptions made at the point of the model design need to be constantly modified and attuned based on the actual state of the operation. Consequently, some research has been conducted on a mechanism to dynamically monitor the trend of changes 
in the model parameters and adapt the simulation model accordingly in real-time (Hammad and Zhang 2011; Lu, Dai, and Chen 2007; Song and Eldin 2012; Akhavian and Behzadan 2012).

On the other hand, new tracking technologies are providing tools for improving productivity and safety by enabling on-site data capturing and decision making using the Global Positioning System (GPS), Real-Time Location Systems (RTLSs), and other geo-positioning technologies (Perkinson, Bayraktar, and Ahmad 2010; Teizer et al. 2010; Peyret et al. 2000; Hildreth, Vorster, and Martinez 2005). These tracking technologies are integrated with 3D design models and Digital Terrain Models (DTMs) in two modes: Automated Machine Guidance (AMG) and Automated Machine Control (AMC). The growing applications of $\mathrm{AMC} / \mathrm{G}$ presents the opportunity to leverage the GPS systems mounted on the construction machines, and the stream of location data flowing therefrom, for purposes other than controlling/guiding machines. This will create an opportunity to use sensory data to accurately capture the impact of simulation parameters (e.g., machine speed, weather, equipment conditions, etc.) that will affect the operations. Nevertheless, not all the data updates suggest that model refinement is required. Given the accuracy of the simulation and the level of detail for which the initial model is designed, only certain types of changes in the environment or simulation parameters may necessitate the refinement of the model. To the best of the authors' knowledge, no research on real-time construction simulation considered a robust filtering mechanism to distinguish non-influential or low-impact data fluctuations from the refinement-worthy changes. As a result, the realism and credibility of real-time simulation as a method to reliably capture the environmental and operational changes tremendously suffer.

The present paper explores a novel approach to the location-aware real-time simulation of earthmoving construction operations with the following three main objectives: (a) to improve the accuracy of real-time simulation through the consideration of environmental parameters that may affect the accuracy of the simulation model, (b) to explore the state-identification rules more in-depth and for all the machines participating in a cyclic operation, and (c) to propose a proactive safety warnings mechanism based on the real-time simulation and pre-defined workspaces.

\section{PROPOSED APPROACH}

As stated earlier, real-time simulation is a concept based on the continuous update of a model initially developed for a particular process. The initial model is inherently established on a large amount of speculations, extracted mainly from similar previous projects. These models require constant adjustment to remain accurate in the face of many anomalies that are rampant in the course of the work execution. The model refinement can be performed using real-time data from the site. In spite of the simplicity of this concept, technical and conceptual challenges for developing a full-fledge framework are manifold. Challenges to be addressed for the development of a real-time simulation include: (a) a simulation tool that can offer the required features for the continuous refinement of the model, (b) a mechanism for data classification and processing to extract meaningful information associated with the models' parameters from raw location data gathered from the site, (c) a method to distinguish the data suggesting major discrepancies between the reality and the model from non-influential fluctuations in the data flow, (d) a framework for adding location awareness to the simulation so that more accurate results can be achieved in view of spatial limitations and possible safety threats.

The structure of the proposed framework for a location-aware real-time simulation is presented in Figure 1. The proposed framework consists of several components including: (a) Location Data Proces-

sor, (b) Rule-based System, (c) Information Filter, (d) Model Analyzer, (e) Model Refiner, (f) Scenario Analyzer, (g) Simulation Engine, and (h) Safety risk analyzer.

\subsection{Location Data Processor}

The raw location data gathered from the site require a multi-step processing before they can be used for data analysis. The processing is required to compensate for the missing or erroneous data. In this frame- 
work, the assumption is that every equipment provides a set of location data that represents several parts of the equipment, e.g., the excavator bucket, body, etc.

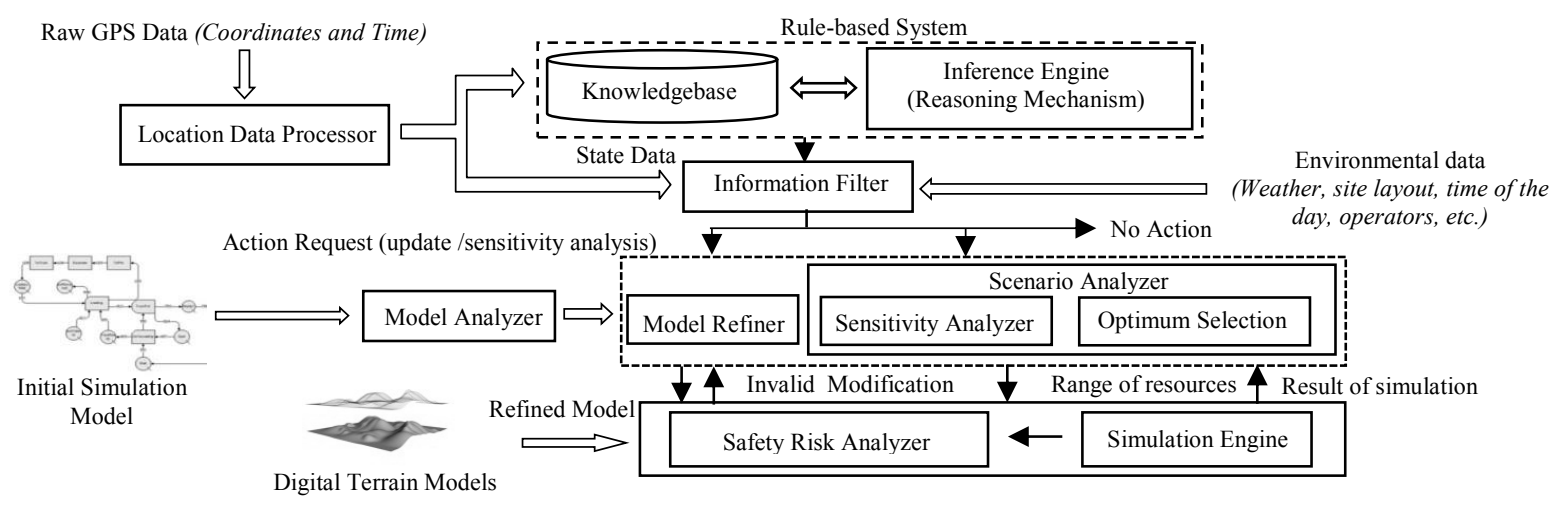

Figure 1: A framework for real-time simulation.

As shown in Figure 2, the data processing includes the following steps: (a) The IDs of different parts of equipment are identified and grouped according to their geometric relationships with respect to the equipment of which they are a part (e.g., location of the body of excavator); (b) The locations of the same parts are averaged over a short period of time, i.e. 1 second; (c) The location data are filtered based on the geometric constraints, i.e. the fixed distance between the parts on an equipment. To determine the acceptable range of error, the distance between the parts are calculated, and using the standard deviation $(\sigma)$ of distances, any readings that fall outside the range of actual distance between two parts $\pm 2 \sigma$ are considered as outliers and deleted; (d) If the error associated with each reading is determined as acceptable from the previous step, then the location data are corrected so that the geometric constrains are satisfied. For instance, as shown in Figure 3(a), if the calculated distance based on the readings of $A_{l}$ and $B_{l}$ is more than what it is measured to be $(L)$, the amount of error is calculated, and the correction is equally distributed between the two parts, resulting in points $A_{1}^{\prime}$ and $B_{1}^{\prime}$; (e) The same filtering and corrections are performed for the operational constraints, e.g., maximum speed. For instance, as shown in Figure 3(b), if the distance between two consecutive readings $A^{\prime}{ }_{1}$ and $A_{2}$ of the same part of a piece of equipment is $L^{\prime}$, while based on the maximum speed of the equipment this distance cannot be more than $L$, a correction is applied to the latter point and the new location is calculated $\left(A_{2}^{\prime}\right)$; and finally (f) If there are any missing data, it will be calculated using interpolation. Once the data is processed and the erroneous data are filtered and corrected, the rules from the knowledge-based system are used to identify the state of the equipment.

\section{$2.2 \quad$ Rule-based System}

The data obtained from the location data processor is not usable unless put under processing. The GPS transmits information about the location of particular objects and the time in which the readings were made. However, for these readings to be transformed into meaningful information usable for the simulation purpose, the location data need to be converted to modeling parameters, e.g., durations and speed. To materialize this, it is required to develop a knowledgebase which encapsulates expert rules that help interpret and transform the purely geographical data. For instance, from an array of GPS readings coming from a receiver attached to a truck, we need to identify when the truck starts traveling to the dumping location or how long it has been in a queue before it is serviced by a loader. Although a similar concept has been proposed before (Song and Eldin 2012), the proposed rules are restricted to one type of equipment, i.e. trucks, and several states, such as out of service, have not been covered. Additionally the discrepancies between the GPS data and the simulation model representing a permanent change have been treated as an assumption, while in the present framework an information filter is applied to sift the transient from the permanent changes. 


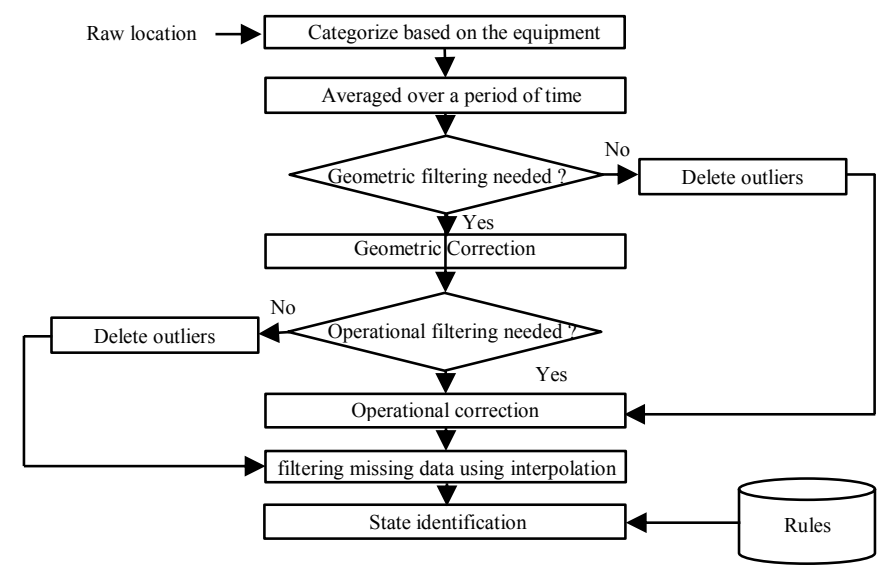

Figure 2: Flowchart of data processing for state identification.

The rule-based system transforms GPS data to simulation parameters and comprises a knowledgebase and a reasoning mechanism. The knowledgebase contains all the rules and heuristics which determine the state of machines and their current phase in the operation. These rules are experience-driven and casedependent. On the other hand, using the knowledgebase, the inference engine helps determine the modeling parameters that are of interest for the simulation.

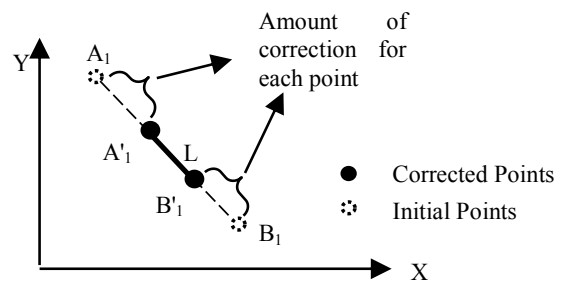

(a) Geometric Constraint Correction

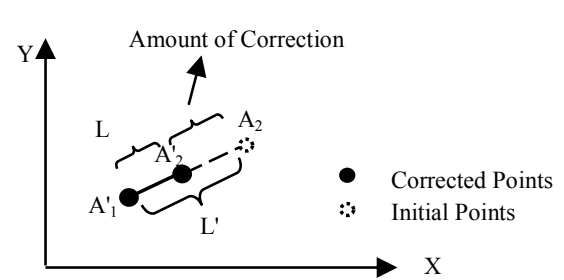

(b) Operational Constraint Correction

Figure 3: Corrections based on the geometric and operational constraints.

Given the vastness and the large scale of construction projects and types of equipment required, it is almost impossible to extract a general mathematical model that performs the transformation of data to the required modeling parameters for all types of projects and machines. Expert rules need to be developed based on the exclusive characteristics of the project, types of machines in the operation and the conditions of the site. The zone-detection is an integral part of these expert rules, especially for equipment whose cyclic operations take place over a geographically scattered area, e.g., trucks. If the approximate area in which a certain activity occurs is known, then it will be possible to associate the coordinates of the equipment to a certain activity. For this purpose, geo-fences can be used to create activity-specific zones. Geo-fences are virtual contours drawn around an area that help detect if a unit has entered a known area (Reclus and Drouard 2009). Although this alone will suffice to infer the initiation of certain types of activities, mainly activities that involve only one resource, when more than one resource need to be engaged for an activity to commence, or when there exists the possibility of the formation of queues, it is required to apply some additional rules. For instance, a truck will be identified in the phase of loading when (a) it is in the loading area and (b) compared to all other trucks in the loading area, it is the closest to the loader. Other determining rules that can be used to convert GPS data to the required information need to be developed based on the modeling parameters of interest and the particular characteristics of the project.

The knowledgebase comprises state-identification rules that are expressed in terms of two types of variables, namely dynamic and constant variables. Constant variables are basically the coordinates of vir- 
tual geo-fences that are marked by the exclusive activities that take place within a zone. Dynamic variables, on the other hand, are auxiliary factors, e.g., distance from the loader, angle of the bed of the truck, etc., that help more accurately calculate the modeling parameters, e.g., duration and speed.

To provide a tangible, a simple hauling-dumping project is used. In this example, a team of two trucks, an excavator and a conveyor belt, as the indicator for the dumping point, are assigned to a hauling task. All equipment are equipped with GPS receivers and identified by unique IDs. For trucks, this simple operation forms four distinct areas, each of which is marked with an exclusive activity. The entrance time and the exit time of each truck to the corresponding area can be determined by the juxtaposition of the truck's GPS data and the geo-fences. The entrance to an area and the duration of stay coupled with the additional conditions, e.g., distance from the loader, help compute the precise durations associated with various activities. For instance, the beginning of the loading activity can be determined by the fulfillment of the combined conditions of the truck being in the loading area, truck's zero velocity and the truck being the closest to the loader. Similarly, the end of the loading is indicated by the increase in the truck's velocity and its distance from the excavator. With the above states identified, the loading time can be calculated through Equation (1):

$$
L D_{i j}=E L T_{i j}-S L T_{i j}
$$

where:

$$
\begin{aligned}
L D_{i j} & =\text { Loading duration of truck } i \text { in cycle } j ; \\
E L T_{i j} & =\text { End of loading time of truck } i \text { in cycle } j ; \\
S L T_{i j} & =\text { Start of loading time of truck } i \text { in cycle } j .
\end{aligned}
$$

In a similar manner, nine different possible states can be identified for a single truck in the above simple example. It is worth mentioning that every state is bounded by start and end events. For instance the state of loading has the start of loading and end of loading events. The inference engine contains the reasoning mechanism that uses the rules within the knowledgebase to infer the corresponding values of the modeling parameters from the incoming flow of GPS data, i.e. time and coordinates.

As for excavators, given the higher Degrees of Freedom (DoFs) of the machines and the finer motions that segregate different states, the state-identifications rules are more sophisticated. Five states can be identified for an excavator in the above-mentioned example when it is stationary, namely under loading, swinging with the full bucket, dumping, swinging with the empty bucket and idle. These states can be identified without zone-detection on the account that the information regarding the velocity of the bucket and the direction of its move suffice for the state-identification of the excavator. Table 1 summarizes the state identification rules for the truck and excavator.

In the case of the excavator, there exists an additional state of repositioning, when the excavator changes its loading position. The identification of this state presents a more complex challenge as it requires pattern recognition so that the travel of the bucket to an unknown new zone can be detected dynamically. Given that the operation of the excavator is highly cyclic with a relatively predictable pattern, and knowing that the repositioning commences with a deviation from the known cyclic pattern (loadswing-dump-swing back) and results in a new cyclic pattern, pattern recognition can help identify the repositioning state. However, this is outside the scope of the current paper.

\subsection{Information Filter}

The information filter monitors various sources, and should it detect discrepancies that demand a change of the model, it will send a request to the Model Refiner or scenario analyzer for further actions. For this component to be able to distinguish refinement-requiring discrepancies from negligible data fluctuation, it is required to collect data from various sources other than GPS. Parameters that may carry transient implications on the process includes, but not limited to, temperature, humidity, precipitations, operators' skill, equipment condition, working hours, time of the day, dynamic changes to the site layout, accidents, etc. While any of the above parameters could affect the location data, it is conceivable that their impacts 
are temporary. Accordingly, every machine state and the pertinent simulation parameters need to be processed and analyzed in line with a set of corresponding environmental data.

Table 1: Identification rules for the truck and excavator's states.

\begin{tabular}{|c|c|c|c|c|}
\hline Equipment & State & $\begin{array}{l}\text { Identified } \\
\text { Zone }\end{array}$ & Dynamic Variables & Identification Rule \\
\hline \multirow{9}{*}{ Truck } & In Loading queue & Loading & $\begin{array}{l}\text { Velocity, Distance to the exca- } \\
\text { vator }\end{array}$ & $\begin{array}{l}\text { The truck is in the loading zone but it is not the closest truck to the } \\
\text { loader and its velocity is zero }\end{array}$ \\
\hline & Waiting for loading & Loading & $\begin{array}{l}\text { Velocity, Distance to the exca- } \\
\text { vator, State of excavator }\end{array}$ & $\begin{array}{l}\text { The truck is in the loading zone, it is the closest to the loader and its } \\
\text { velocity is zero but the excavator is not in dumping }\end{array}$ \\
\hline & Loading & Loading & $\begin{array}{l}\text { Velocity, Distance to the exca- } \\
\text { vator, State of excavator }\end{array}$ & $\begin{array}{l}\text { The truck is in the loading zone, it is the closest to the loader, its } \\
\text { velocity is zero and the excavator is dumping }\end{array}$ \\
\hline & Hauling & Hauling & Velocity & The truck is in the hauling zone and its velocity is not zero \\
\hline & Out of service & Hauling & Velocity & The truck is in the hauling zone and its velocity is zero \\
\hline & Dumping & Dumping & $\begin{array}{l}\text { Velocity\& Distance to the } \\
\text { Conveyer }\end{array}$ & $\begin{array}{l}\text { The truck is in the dumping zone., it is the closest to the conveyor } \\
\text { belt, and its velocity is zero }\end{array}$ \\
\hline & $\begin{array}{l}\text { In the dumping } \\
\text { queue }\end{array}$ & Dumping & $\begin{array}{l}\text { Velocity\& Distance to the } \\
\text { Conveyer }\end{array}$ & $\begin{array}{l}\text { The truck is in the dumping zone, it is not the closest unit to the } \\
\text { conveyor belt and its velocity is zero }\end{array}$ \\
\hline & Returning & Returning & Velocity & The truck is in return zone and its velocity is not zero \\
\hline & Out of service & Returning & Velocity & The truck is in the return zone and its velocity is zero \\
\hline \multirow{5}{*}{ Excavator } & Under loading & N/A & Velocity \& Motion direction & $\begin{array}{l}\text { The bucket is predominantly moving in a vertical plane with a low } \\
\text { velocity }\end{array}$ \\
\hline & Swinging (loaded) & N/A & $\begin{array}{l}\text { Motion direction \& Distance to } \\
\text { the truck }\end{array}$ & $\begin{array}{l}\text { The bucket is predominantly moving in a horizontal plane and it is } \\
\text { moving toward the truck }\end{array}$ \\
\hline & Dumping & N/A & $\begin{array}{l}\text { Motion direction \& Distance to } \\
\text { the truck }\end{array}$ & $\begin{array}{l}\text { The bucket is relatively stationary and its location intersects with the } \\
\text { truck's bed }\end{array}$ \\
\hline & Swinging (empty) & $\mathrm{N} / \mathrm{A}$ & $\begin{array}{l}\text { Distance to the truck \& Motion } \\
\text { direction }\end{array}$ & $\begin{array}{l}\text { The bucket is predominantly moving in a horizontal plane and it is } \\
\text { moving away from the truck }\end{array}$ \\
\hline & Idle & N/A & Velocity & The bucket is not moving \\
\hline
\end{tabular}

\subsection{Model Analyzer}

The Model Analyzer interprets the initial model and translates the simulation's internal logic to a formalized and modifiable format. It then disintegrates the model into the constituent components and formalizes the causal relationships, activities and resources so that they can be individually modified and reassembled by the Model Refiner and scenario analyzer for further execution.

\subsection{Model Refiner}

The input for this component is the change request coming from the information filter, representing a major deviation from the existing values in the model, the state of equipment resulting from the rule-based system and the parsed model coming from the model analyzer. The Model Refiner identifies the parameters that need to be adjusted based on the change request placed by the information filter and updates the model accordingly.

\subsection{Scenario Analyzer}

The scenario analyzer mainly performs sensitivity analysis over a range of resource quantities to help optimize the configuration of the fleet. For example, if a unit falls out of service, this component would allow the management to explore the possible options they can choose from. This component includes an engine for the sensitivity analysis and a module to opt out the most optimum solution.

\subsection{Simulation Engine}

The simulation engine is a platform on which the process model is run and the subsequent report is generated. This component goes through the model simulation and flows the resources amid the network of activities to perform the time and productivity evaluation of the model. However, the selection of the opti- 
mum solution is done once all the scenarios are validated by the safety risk analyzer component regarding safety requirements.

\subsection{Safety Risk Analyzer}

This component is responsible for the identification of the potential safety risks through the integration of planning and scheduling data with the real-time location data. Briefly, every task is marked by Dynamic Virtual Fences (DVFs) that outlines the geometric boundaries within which the task is expected to be executed. The dynamism of the DVFs is due to the fact that they will be updated as the project progresses along the project schedule. This component ensures that all equipment remain within their designated workspace all the time and triggers a warning if necessary. More elaborate discussion of this component can be found in Setayeshgar et al. (2013).

\section{PROTOTYPE DEVELOPMENT}

To demonstrate the applicability of the proposed framework, a prototype application was developed and tested in this research. Of all the components enumerated in Section 2, location data processor, rule-based system, Model Analyzer and Model Refiner were chosen and developed separately.

The Location data processor and rule-based system are, for time being, developed in Microsoft Excel environment. The earlier receives the raw location data as input and processed them through the steps elaborated in Section 2.1. Once the location data are flittered and trimmed, the rule-based system considers many different location parameters to infer the state of different pieces of equipment, as explained in Section 2.2, and presents them in a different sheet. The full integration and automation of these two components requires more effort.

The Model Analyzer and Model Refiner is developed in Microsoft Visual Basic for Application (VBA) platform embedded in Microsoft Excel. Stroboscope (Martinez 1996) is chosen as the simulation engine due to its palpable strengths in extensibility, robustness, availability, compatibility with simulation of construction operations and the ease of use. The main scope of the prototype, at the current stage of the research, is to implement the Model Analyzer and Model Refiner and integrate them with a simulation engine. In the proposed framework, once the raw GPS data are processed in the rule-based system, and thus transformed to information about the machine state, and then filtered by the information filter, they are either sent to the Model Refiner or scenario analyzer. However, at the current stage of the prototype, the assumption is that the input GPS coming from the rule-based is already classified by the information filter as influential changes requiring the refinement of the simulation model.

The structure of the Model Refiner is illustrated in Figure 4. The initial simulation model is fed to the prototype, and the Model Analyzer parses the model and creates the list of durations and features that are used in the model. At this point, the user is asked to choose the variables and parameters that are used in the real-time simulation, e.g., hauling speed, and correlate the chosen variables/parameters with the relevant state data coming from the information filter. For instance, the user determines that the actual speed of truck 1 in the hauling state is the subtraction of state data annotated B and A, i.e. column B and A in an Excel sheet. To establish this correlation, the user choose the hauling speed in the interface of the realtime simulation module and define the new value as (B-A). It is worth noting that the investigation of how this process can be automated is part of the future work of this research. Once the correlations are made, the prototype monitors the flow of state data and updates the model according to the incoming state information. Every time an update is made, the model is re-run in the simulation model and results are published in the Excel sheet.

\section{CASE STUDY}

The following case study aims to validate how the state of the equipment can be derived from location and sensory data and further used for the decision making and real-time simulation. Although the imple- 
mentation of a fully integrated system is beyond the scope of the present research, the developed prototype is used to test the proposed approach.

The case study demonstrates the tasks related to the following components of the framework: (a) Location data processor: filtering and correcting the location data so that data errors are minimized, (b) Rule-based system: inferring the state and pose information from the location using the rules from Table 1, and (c) Model Refiner: updating the simulation model using the developed Model Refiner.

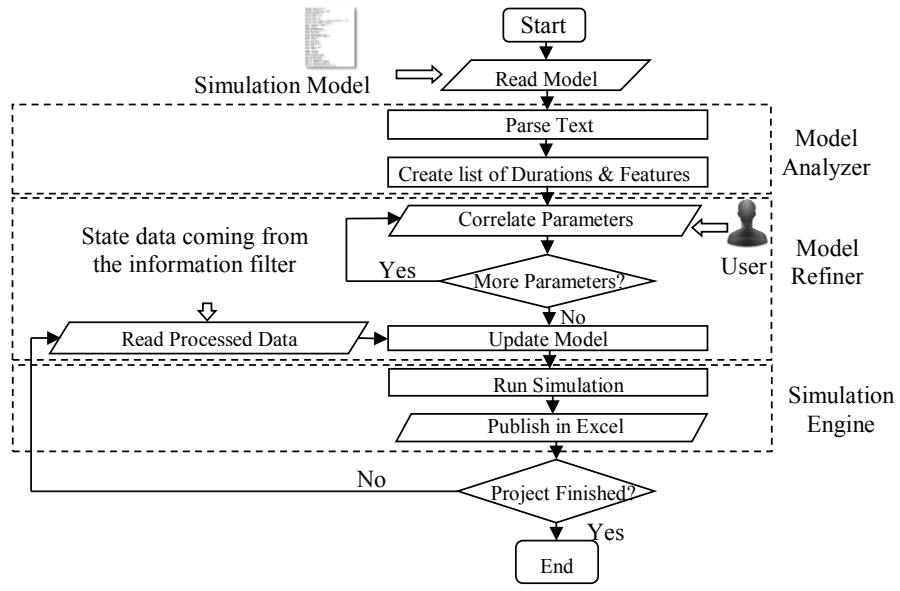

Figure 4: The process flow of the Model Refiner.

\subsection{Setting of the Test}

A test was carried out in the laboratory environment, where two radio-controlled (RC) machines, one 1/24 scaled model truck, and one 1/12 model excavator (Hobby Engine, 2013), were utilized to simulate a simple loading-hauling-dumping operation. The reason for selecting this environment is that it can be fully controlled and can be repeated as many times as necessary. The two pieces of equipment are equipped with tags of an Ultra-Wideband (UWB) Real time Location System (RTLS) (Ubisens 2013), which can provide the location information of these tags similar to GPS receivers in the case of AMC/G. The application of UWB in construction sites has been investigated by multiple researchers (Saidi and Lytle 2008; Giretti et al. 2009; Teizer et al. 2010; Zhang et al.2012). These studies have demonstrated the potentials of UWB as a reliable tracking technology with an acceptable level of accuracy that allows its application for both indoor and outdoor construction work.

The case study covered four full cycles of loading and hauling, which consisted of loading, hauling, dumping and returning operation for the truck and the loading, swinging, dumping, swinging back and repositioning operations for the excavator. Zones within which each of the afore-mentioned operation takes place were marked and their corresponding coordinates were determined. These zones later establish the base for the state-identification of the equipment. Figure 5 shows the setting of the case study which contains four main parts, namely, the excavation area, loading area, hauling area and the dumping area. The truck is loaded in the loading area by the excavator; it moves to the dumping area and dumps its load. On the other hand, the excavator obtains a load from the borrow pit, swings to the loading area, dumps the material into the truck and swings back. The excavation area is divided into two work spaces. After two cycles of the truck, the excavator repositions to a new work space within the same excavation zone, which is marked by WS2 in Figure 5(a), and continues to load the truck for two more cycles. A five-minute test was performed in which 4 cycles of loading-and-dumping were simulated. Figure 5(b) depicts the test environment, the site layout, the zoning and different UWB tags attached to different parts of the equipment. Four UWB tags were assigned to the excavator and two were assigned to the truck in order monitor their movement. Sensors are installed at the locations providing the best coverage for the 
area. The slot interval was set to 16 which is equivalent to 9.61 readings per second. However, after the analysis of the test results, the actual average update rate was determined to be 8 readings per second.

On the specifications of the equipment used in the case study, the truck has two motors that allow the movement of the body (drive forward/backward, turn right/left) and the bed of the truck (up/down). The excavator has five motors that allow the movement of the body (drive forward/backward, turn right/left) and the boom (moving forward/backward and up/down). Both models can be manually controlled using a remote control with different buttons and joysticks that allow the movement of one DoF at a time.

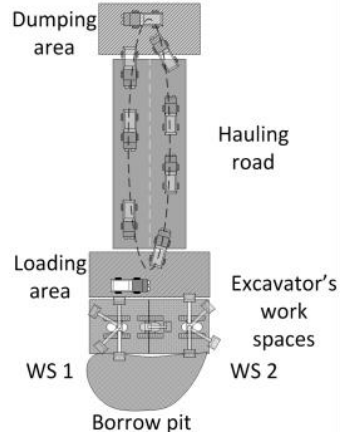

(a) 2D model of the case study

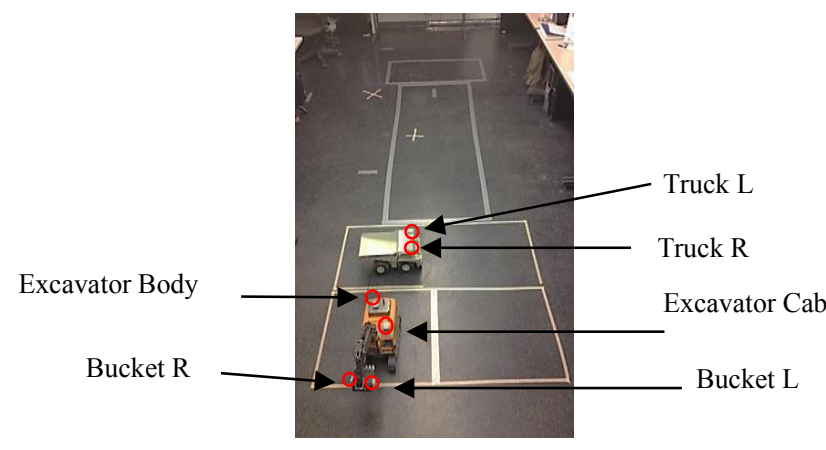

(b) A picture of the actual case study, UWB tags are marked on the picture

Figure 5: The zoning and layout of the case study.

\subsection{Results and Discussions}

The location data processor, explained in Section 2.1, is used to process the location data. The effect of the data processing on the traces of movement for different tags is shown in Figure 6.

Table 2 shows an excerpt of the result of applying the state identification rules. Figure 7 shows the results of the state identification for the entire test. Dotted lines in Figure 7 represent the transition between two adjacent states and the continuous lines indicate the continuity of the equipment's presence in the corresponding states. The unrealistic ratio of different states in relation to one another, e.g., the hauling time to waiting time for the loading, can be ascribed to the combined effects of the scale of the equipment and site together with the equipment's operational speed. Also, given that only one truck was utilized in this test, states that involve waiting in queues are not pertinent to this case study.

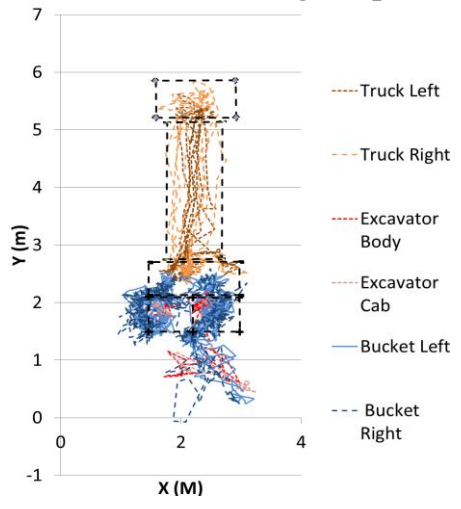

(a) Before data processing

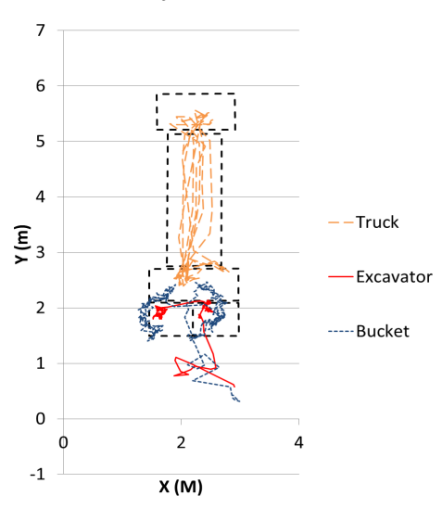

(b) After data processing

Figure 6: Operation's processed data. 
The above-mentioned results are used to update the simulation model. Although the developed Model Refiner prototype is designed to automatically update the model based on the flow of sensor data, given that the connection between the information filter and the Model Refiner is not yet established at the current state of the research, the Model Refiner prototype is applied off-line. This means that the results of the rule-based system were manually transferred to the Model Refiner.

Table 2: Excerpt of the state-identification for the truck.

\begin{tabular}{|l|l|l|l|l|l|}
\hline Equipment & Time (s) & Zone & Distance to the Excavator $(\mathbf{m})$ & Speed of truck (m/s) & \multicolumn{1}{c|}{ State } \\
\hline Truck & 59 & Loading & 0.36 & 0.02 & Waiting for loading \\
\hline Truck & 61 & Loading & 0.25 & 0.00 & Under Loading \\
\hline Truck & 64 & Hauling & 0.90 & 0.55 & Hauling \\
\hline Truck & 68 & Dumping & 3.35 & 0.47 & Dumping \\
\hline
\end{tabular}

The simulation parameters defined in Section 4.2 is updated based on the average values for the duration of different activities from the test as shown in Table 3 . The service time of the excavator is assumed to remain the same. The results suggest that the actual productivity of the operation is $4.6 \%$ less than assumed which is also manifested in a longer total duration of 13 seconds for the 4-cycle operation.

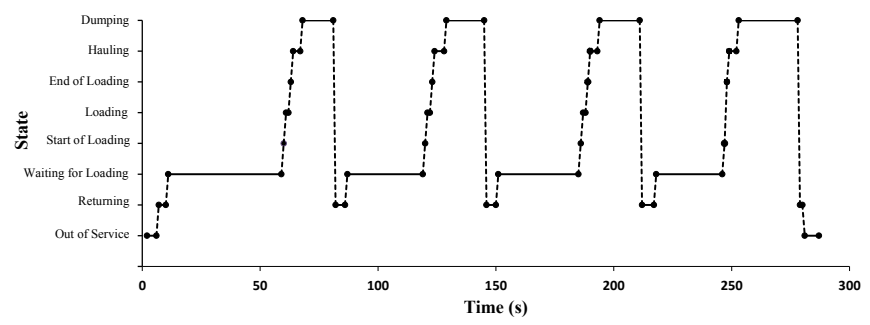

Figure 7: The transition of the state of the truck through the test.

Similarly, as explained in Section 2.8, the above results can be used for safety purposes. For instance, if the truck leaves the designated zones within which it is expected to work, this deviation can be identified and safety warnings are triggered. Also, if two equipment that have a certain proximity threshold, i.e. they are not supposed to work closer than a certain distance, trespass the threshold, a safety warning can be triggered, and subsequently, using $\mathrm{AMC} / \mathrm{G}$ an immediate action can be taken, e.g., stopping the equipment.

Table 3: Simulation parameters values as assumed (a) and measured in real-time (b).

\begin{tabular}{|l|c|c|c|c|c|c|}
\hline \multirow{2}{*}{ Simulation Parameters } & \multicolumn{2}{|c|}{ Duration (s) } & \multicolumn{2}{c|}{ Productivity (truck/min) } & \multicolumn{2}{c|}{ Total Duration (s) } \\
\cline { 2 - 7 } & Before & After & Before & After & Before & After \\
\hline Excavator service time & 60 & 60 & & & & \\
\hline Loading time & 2 & 3.50 & \multirow{2}{*}{0.9} & \multirow{2}{*}{0.86} & 267 & 280 \\
\hline Hauling time & 6 & 4.25 & & & \\
\hline Dumping & 10 & 16.33 & & & & \\
\hline Returning & 3 & 5 & & & & \\
\hline
\end{tabular}

\section{CONCLUSIONS}

The present paper explored the concept of location-aware real-time simulation of earthmoving projects through the development of a framework and the implementation of a prototype. The proposed framework, encapsulating the components for dynamic filtering and 4D modeling, offers a solution for monitoring simulation models in relation to real-time data from the site and refining the model if any major discrepancies are observed. Four components of the proposed system, namely location data processor, rule- 
based system, Model Analyzer and Model Refiner, were developed as a prototype and implemented in a case study to examine the feasibility of the proposed method.

A case study was designed and implemented to: (a) validate the functionality of the developed prototype; and (b) investigate how real-time location data can be transformed into meaningful information pertinent to the simulation model and further used as the basis for the generation of state-identification rules.

The proposed framework offers a trifold enhancement to the real-time simulation frameworks proposed by other scholars: (a) A filtering module is proposed that can help distinguish the transient environmental changes with minimal long-term impacts on the productivity from the influential changes that will greatly impact an operation, (b) the safety risk analyzer module is incorporated in the system to identify the potential hazardous situation that might be borne in the face of the changing environment, and (c) the state-identification rules were explored beyond what is proposed by other researchers, incorporating rules about the operation of excavators.

In spite of the primary success of the developed prototype, further efforts are required to complement the prototype so that it covers all aspects of the proposed framework. Firstly, a wider range of construction machines and operations needs to be investigated to create a comprehensive rule-based system for state identification. Also, the applicability of advanced soft computing methods, e.g., neural network, for state-identification needs to be scrutinized. Secondly, the information filter component needs to be developed and tested. Thirdly, although 4D modeling and visualization was briefly tested, its full integration with real-time simulation has to be further addressed. Finally, all the components need to be combined and tested against an actual case study for the evaluation of the actual benefits the framework can offer in terms of more realistic representation of construction operations.

\section{REFERENCES}

AbouRizk, S. 2010. "Role of Simulation in Construction Engineering and Management." Journal of Construction Engineering and Management 136 (10): 1140-1152.

Akhavian, R., and A. H. Behzadan. 2012. "An Integrated Data Collection and Analysis Framework for Remote Monitoring and Planning of Construction Operations." Advanced Engineering Informatics 26 (4): 749-761.

Dawood, N., and Z. Mallasi. 2006. "Construction Workspace Planning: Assignment and Analysis Utilizing 4D Visualization Technologies." Computer-Aided Civil and Infrastructure Engineering 21 (7): 498-513.

Doriani, A., M. Mawlana, and A. Hammad. 2013. "Simulation-Based Deterministic and Probabilistic 4D Modeling for Planning and Scheduling of Elevated Urban Highway Reconstruction Projects." Transportation Research Board (TRB). Washington D.C.: Transportation Research Board (TRB) Conference.

Giretti, A., A. Carbonari, B. Naticchia, and M. De Grassi. 2009. "Design and first development of an automated real-time safety management system for construction sites." J. Civil Eng. Manage. 15 (4): 325-336.

Halpin, D. W., and L. H. Martinez. 1999. "Real World Applications of Construction Process Simulation. " In Proceeding of the 1999 Winter Simulation Conference: Simulation---a bridge to the future, Edited by P. A. Farrington, H. B. Nembhard, D. T. Sturrock, and G. W. Evans, 956 - 962. Piscataway, New Jersey: Institute of Electrical and Electronics Engineers, Inc.

Hammad, A., and C. Zhang. 2011. "Towards Real-Time Simulation of Construction Activities Considering Spatio-Temporal Resolution Requirements for Improving Safety and Productivity." In Proceeding of the 2011 Winter Simulation Conference, edited by S. Jain, R.R. Creasey, J. Himmelspach, K.P. White, and M. Fu, 3533-3544. Piscataway, New Jersey: Institute of Electrical and Electronics Engineers, Inc.

Hassan, M. M., and S. Gruber. 2008. "Simulation of Concrete Paving Operations on Interstate-74." Journal of Construction Engineering and Management 134 (2): 2-9. 
Hassan, S., M. Al-Hussein, and P. Gillis. 2010. "Advanced Simulation of Tower Crane Operation Utilizing System Dynamics Modeling and Lean Principles." In Proceeding of the 2010 Winter Simulation Conference, edited by B. Johansson, S. Jain, J. Montoya-Torres, J. Hugan, and E. Yücesan, 3262-3271. Piscataway, New Jersey: Institute of Electrical and Electronics Engineers, Inc.

Hildreth, J., M. Vorster, and J. Martinez. 2005. "Reduction of Short-interval GPS Data for Construction Operations Analysis." Journal of Construction Engineering and Management 131 (8): 920-927.

Hobby Engine. (2013). http://www.hobbyengine.com.hk/catalog/product.php?cid=31

Lu, M., F. Dai, and W. Chen. 2007. "Real-time Decision Support for Planning Concrete Plant Operations Enabled by Integrating Vehicle Tracking Technology, Simulation, and Optimization." Canadian Journal of Civil Engineering 34 (8): 912-922.

Martinez, J. C. 1996. "STROBOSCOPE State and Resource Based Simulation of Construction Processes. " Ph.D. thesis, University of Michigan.

Perkinson, C. L., M. E. Bayraktar, and I. Ahmad. 2010. "The Use of Computing Technology in Highway Construction as a Total Jobsite Management Tool." Automation in Construction 19(7): 884-897.

Peyret, F., J. Jurasz, A. Carrel, E. Zekri, and B. Gorham. 2000. "The Computer Integrated Road Construction Project." Automation in Construction 9(5): 447-461.

Reclus, F., and K. Drouard. 2009. "Geofencing for Fleet \& Freight Management." In the Proceedings of 9th International Conference on Intelligent Transport Systems Telecommunications (ITST), 353-356.

Saidi, K., and A. Lytle, 2008. "NIST Research in Crane Automation: 2007 Overview." 87th Annual Meeting, Transportation Research Board.

Setayeshgar, S., A. Hammad, F. Vahdatikhaki, and C. Zhang. 2013. "Real Time Safety Analysis of Construction Projects Using BIM and RTLS." The 30th International Symposium on Automation and Robotics in Construction and Mining (ISARC). Montreal, Canada.

Song, L., and N. N. Eldin. 2012. "Adaptive Real-Time Tracking and Simulation of Heavy Construction Operations for Look-Ahead Scheduling." Automation in Construction 27: 32-39.

Teizer, J., B. S. Allread, C. E. Fullerton, and J. Hinze. 2010. "Autonomous Pro-Active Real-Time Construction Worker and Equipment Operator Proximity Safety Alert System." Automation in Construction 19 (5): 630-640.

Ubisense. (2013). http://www.ubisense.net/en/

Zayed, M. T., and D. Halpin. 2001. "Simulation of Concrete Batch Plant Production." Journal of Construction Engineering and Management 127 (2): 132-141.

Zhang, C., A. Hammad, and S. Rodriguez, 2011. "Crane Pose Estimation Using UWB Real-Time Location System. " Journal of Computing in Civil Engineering 26 (5): 625-637.

\section{AUTHOR BIOGRAPHIES}

Faridaddin Vahdatikhaki is a PhD student in Building, Environmental and Civil Engineering Department (BCEE) at Concordia University. His research interests are automation in construction, simulation in construction, and Knowledge, management systems. His email address is f_vahdat@encs.concordia.ca.

AMIN HAMMAD is a Professor at the Concordia Institute for Information Systems Engineering. His research focuses on sustainable infrastructure lifecycle management systems and investigates several methods and techniques including spatio-temporal information modeling and analysis, simulation, visualization, optimization, wireless communications, sensing, auto-identification, and real-time location tracking. His email is hammad@ciise.concordia.ca.

SHAYAN SETAYESHGAR is a Master student in the Building, Civil and Environmental Engineering Department at Concordia University. His research areas are building information modeling, real-time location systems, and IT applications in construction. His email address is m_setaye@encs.concordia.ca. 